\title{
Chronometric Electrical Stimulation of Right Inferior Frontal Cortex Increases Motor Braking
}

\author{
Jan R. Wessel, ${ }^{1 \star}$ Christopher R. Conner, ${ }^{2 \star}$ Adam R. Aron, ${ }^{1}$ and Nitin Tandon ${ }^{2,3}$ \\ ${ }^{1}$ Psychology Department, University of California, San Diego, La Jolla, California 92093, and ${ }^{2}$ Department of Neurosurgery, University of Texas Medical \\ School at Houston, and ${ }^{3}$ Memorial Hermann Hospital, Texas Medical Center, Houston, Texas 77030
}

\begin{abstract}
The right inferior frontal cortex (rIFC) is important for stopping responses. Recent research shows that it is also activated when response emission is slowed down when stopping is anticipated. This suggests that rIFC also functions as a goal-driven brake. Here, we investigated the causal role of rIFC in goal-driven braking by using computer-controlled, event-related (chronometric), direct electrical stimulation (DES). We compared the effects of rIFC stimulation on trials in which responses were made in the presence versus absence of a stoppinggoal ("Maybe Stop" [MS] vs "No Stop" [NS]). We show that DES of rIFC slowed down responses (compared with control-site stimulation) and that rIFC stimulation induced more slowing when motor braking was required (MS) compared with when it was not (NS). Our results strongly support a causal role of a rIFC-based network in inhibitory motor control. Importantly, the results extend this causal role beyond externally driven stopping to goal-driven inhibitory control, which is a richer model of human self-control. These results also provide the first demonstration of double-blind chronometric DES of human prefrontal cortex, and suggest that—in the case of rIFC—-this could lead to augmentation of motor braking.
\end{abstract}

Key words: inhibitory control; direction electrical stimulation; electrocorticography; stop-signal task; cognitive control

\section{Introduction}

The right inferior frontal cortex (rIFC) is critical for rapidly stopping manual responses when a signal occurs (Aron et al., 2003; Chambers et al., 2006; Verbruggen et al., 2010; Zandbelt et al., 2013b), along with its associated functional and structurally connected network (Aron et al., 2007; Neubert et al., 2010; Forstmann et al., 2012). Although externally triggered stopping is occasionally required in everyday life, a more common requirement is to inhibit a motor tendency according to one's goal. This can be modeled by examining how people slow down when they might have to stop. Indeed, the rIFC is active when subjects anticipate that they might have to stop (Chikazoe et al., 2009; Jahfari et al., 2010; Jahfari et al., 2012; Swann et al., 2013; Zandbelt et al., 2013a), with the level of activation corresponding to the degree of motor caution (Jahfari et al., 2010) and the timing of activation corresponding tightly to the motor response (Swann et al., 2013). Although these findings are consistent with rIFC having a braking function (along with its network), causal evidence is required. To

Received July 30, 2013; revised Nov. 4, 2013; accepted Nov. 6, 2013.

Author contributions: J.R.W., A.R.A., and N.T. designed research; J.R.W. and C.R.C. performed research; J.R.W. and C.R.C. analyzed data; J.R.W., C.R.C., A.R.A., and N.T. wrote the paper.

*J.R.W. and C.R.C. contributed equally to this work.

This work was supported by National Institutes of Health Center for Clinical and Translational Sciences (Grant KL2 RR0224149 to N.T.), the Mischer Neuroscience Institute at Memorial Hermann Hospital, the Keck Center of the Gulf Coast Consortia on the Training in Theoretical and Computational Neuroscience, and the National Institute of Biomedical Imaging and Bioengineering (Grant T32EB006350 to C.R.C.). We thank the patients for volunteering; the nurses and technicians in the epilepsy-monitoring unit at Memorial Hermann Hospital; Jeremy Slater, Giri Kalamangalam, and Omotola Hope for referring these patients for epilepsy surgery; and Nicole C. Swann, Jordan A. Comins, and two anonymous reviewers for helpful comments.

Correspondence should be addressed to Jan R. Wessel, University of California, San Diego, Psychology Department, 3133 McGill Hall, 9500 Gilman Drive, La Jolla, CA 92093. E-mail: jwessel@ucsd.edu.

DOI:10.1523/JNEUROSCI.3468-13.2013

Copyright $\odot 2013$ the authors $\quad 0270-6474 / 13 / 3319611-09 \$ 15.00 / 0$ establish a potential causal role for the rIFC (and/or its connected network) in braking, we used direct-electrical stimulation (DES; Lüders et al., 1995; Borchers et al., 2012; Filevich et al., 2012; Desmurget et al., 2013). We implemented a novel computercontrolled, double-blind stimulation paradigm in which a single pair of DES pulses was time locked to impending movement and functionally localized to rIFC.

We studied four subjects with subdural electrodes implanted for electrocorticography (ECoG). First, in each subject, we localized an rIFC electrode with an "inhibitory-control" ECoG signature during a stop-signal task (SST; Fig. 1A). We screened all electrodes for typical stopping-related activity-patterns (i.e., an increase in beta-band activity; Swann et al., 2009) and/or an increase in gamma-band activity (Swann et al., 2012; 2013). All subjects showed one or both of these signatures in an rIFC electrode (Figs. 2, 3). This, along with an adjacent electrode (bipolar configuration), was designated as the stimulation site for the braking task, which consisted of alternating blocks of "Maybe Stop" (MS) or "No Stop" (NS) trials (Fig. 1B). Based on a recent study showing that rIFC is active around the motor response on MS trials (Swann et al., 2013), we delivered stimulation using a dynamic reaction time (RT) forecast in each condition. Stimulation trials (STIM) and no stimulation trials (SHAM) were delivered alternately.

We predicted that rIFC stimulation would slow down responses compared with sham. In addition, we investigated the difference in stimulation-induced slowing between the MS and NS conditions. If stimulation only induces slowing in the MS condition, this would imply that rIFC-induced braking is context sensitive (Swann et al., 2013; Chiu and Aron, 2013), whereas if stimulation induces slowing in both conditions, then rIFC exerts its braking influence regardless of context. In addition, to estab- 
lish specificity to rIFC, we also performed stimulation of a control site in the temporal lobe in a subset of patients.

\section{Materials and Methods}

Subjects. Four patients undergoing seizure screening for intractable epilepsy volunteered to participate in the study. Two patients were right handed, one was left handed, and one was ambidextrous (this patient used the left hand to perform the task). Patients were 49, 42, 25, and 29 years of age. Two patients were female and two were male. The average IQ was 105.25 (range: $88-118$ ). The study was approved by the ethics committees of the University of Texas (UT) Houston Medical School (IRB \#HSC-MS06-0385) and UCSD (\#131444X).

Procedure. Each subject performed the stopsignal (localizer) task first, during which ECoG data were recorded from all available electrode sites. The data were collected at UT Houston and then sent to analysis at University of California-San Diego. There, the data were quickly analyzed for each channel individually by a rater (J.R.W.) who was blind to the electrode locations to pick an electrode with the typical electrophysiological pattern observed in the SST (greater stop-signal-locked betaband power before stop-signal reaction time [SSRT] for successful compared with failed Stop trials, Swann et al., 2009; increased gamma-band activity for Stop trials, Swann et al., 2012, 2013). Because an electrode selection had to be made within $2-3 \mathrm{~h}$ after localizer data collection, full-frequency event-related spectral perturbation (ERSP) plots could not be obtained for all channels to make a determination of which electrode to stimulate. Therefore, we opted to plot the average power over the entire frequency band of interest (beta: $13-30 \mathrm{~Hz}$; gamma: 30-200 Hz) for both contrasts. The resulting plots on which the electrode selection was based can be seen in Figure 2. The gamma signature was most prevalent. Although only one patient showed the beta signature of interest when the quick analysis was run, subsequent generation of full-spectrum beta plots did reveal the typical beta signature for all patients (Figure 3 ).

The selection procedure resulted in $2-5$ electrodes for each of the 4 patients. These electrode sites were then sent back to UT Houston, where electrode locations were unblinded and 1 of the $2-5$ preselected electrodes was chosen based on proximity to rIFC. In all four subjects, at least one of these electrodes lay completely within the caudal inferior frontal gyrus, albeit at varying ventral/dorsal positions (Fig. 2). This slight variation in position is not unexpected given the considerable variation in IFC anatomy between subjects and the fact that cytoarchitecture is not well reflected by sulcal boundaries (Amunts et al., 1999). Because stimulation was done in a bipolar manner, an adjacent electrode was selected based on anatomical considerations based on proximity to rIFC.

In the two cases with temporal lobe control-site stimulation, the two control-site electrodes were selected purely based on anatomical considerations (for S2) or on anatomical considerations and the stop-taskrelated electrophysiology (for S3; see Results for details).

Stimulation was then done for the braking task. In the patients with control-site stimulation, stimulation sites alternated in a blockwise fashion; that is, sites were switched every 50 trials of the task. This required physically switching the connectors on the stimulator, obviating full computer-controlled trial-by-trial randomization or alternation of stimulation sites. However, we made sure that whereas the site selection was done by the neurosurgeon (N.T.), the experiment was run by a separate experimenter (C.R.C.) who was blind to which site was stimulated on each block.

Localizer task ("stop signal"). At the beginning of each trial, a fixation cross appeared in the center of the screen for $500 \mathrm{~ms}$. Subsequently, a leftor rightward arrow (Go stimulus) appeared on the screen. Subjects had to respond as fast and accurately as possible with the dominant hand (right hand in S1 and S3, left hand in S2 and S4) using the arrow keys on a computer keyboard. Subjects had $1500 \mathrm{~ms}$ to respond to the Go stimulus. Overall trial duration was fixed at $3000 \mathrm{~ms}$, with unused time going to the intertrial interval. On $33 \%$ of trials, a stop signal occurred $(900 \mathrm{~Hz}$ sine-wave tone, $250 \mathrm{~ms}$ duration) at a delay after the Go stimulus (stopsignal delay [SSD]). The SSD was dynamically adjusted to achieve an overall probability of stopping of 50\%: In case of a successful stop, the current SSD was prolonged by $50 \mathrm{~ms}$; in case of a failed stop, it was shortened by $50 \mathrm{~ms}$. Individual SSD staircases were used for left- and rightward Go stimuli. Before the ECoG recording session, a gauging session (80 trials) was performed using the same paradigm to gain a proper SSD estimate for the recording session. For this gauging session, SSD was initially set to $200 \mathrm{~ms}$. For the ECoG recording session, the SSD estimates from the gauging session were used. In the ECoG recording session, 300 trials were performed.

ECoG recordings. ECoG data were collected at $2000 \mathrm{~Hz}$ (bandwidth 0.1-750 Hz) using the NeuroPort recording system (Blackrock Microsystems). ECoG data were visually inspected for interictal epileptiform discharges and electrical noise. Electrodes were referenced to a common average of all electrodes except for those with $60 \mathrm{~Hz}$ noise or epileptiform activity when initially referenced to an artificial 0 (Conner et al., 2011). To avoid including any brain regions with potentially abnormal physiology, all electrodes that showed interictal activity (spikes) or that were 


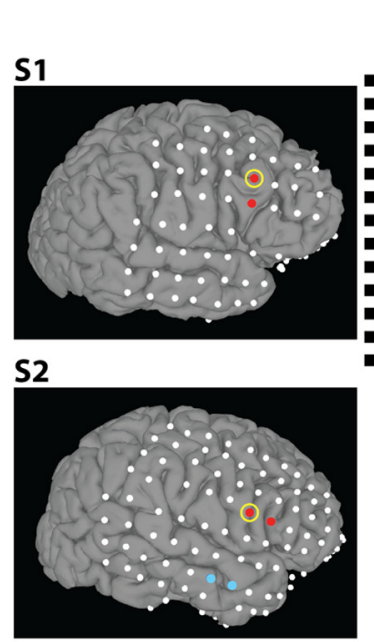

S3

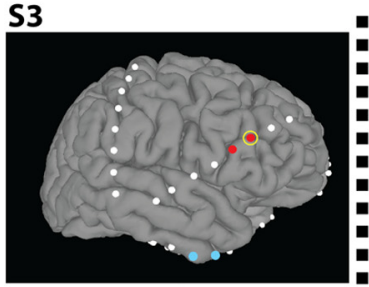

S4

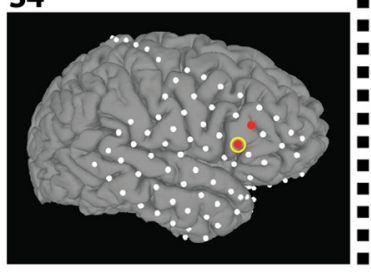

Gamma-band power Locked to go stimulus
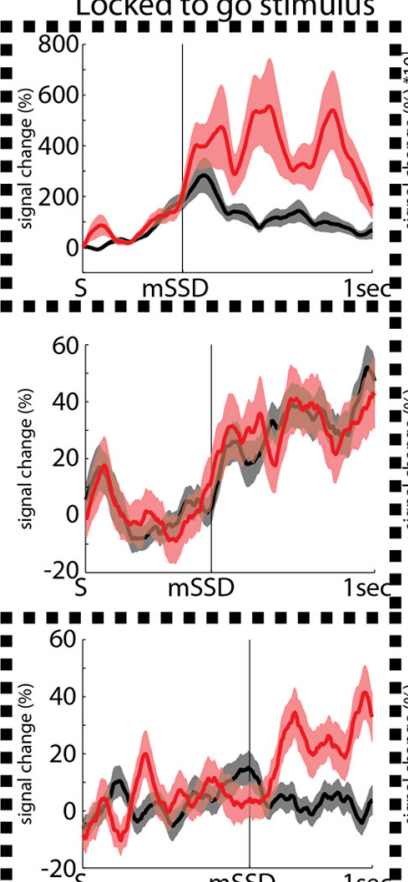

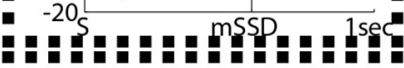

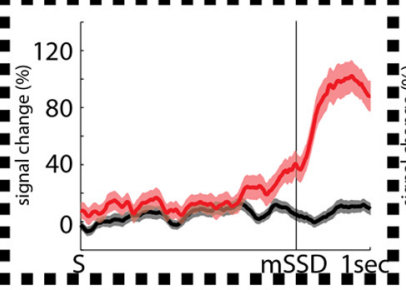

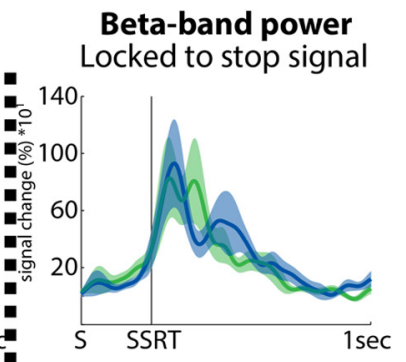

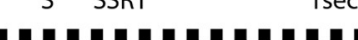

-
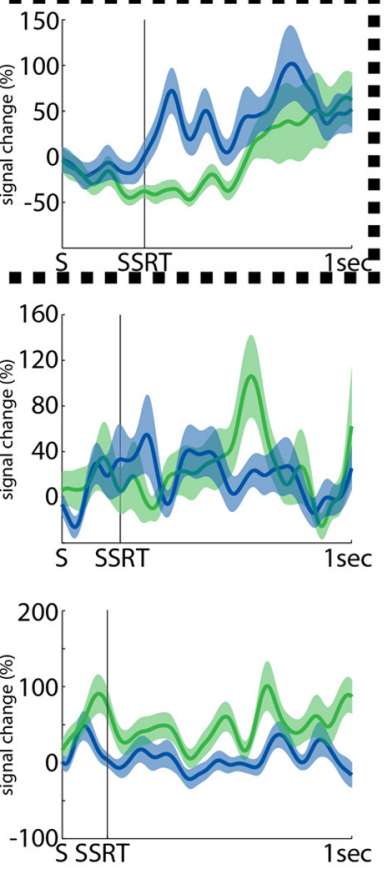

rIFC site, positive pole (localized)

rIFC site, negative pole control site
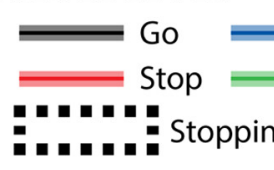

Stopping-related signature

Figure 2. ECOG data from the localizer SST in all four patients. Left, Electrode coverage for the individual patients and the respective locations of the experimental and control sites (no control site in S1 and S4) that were stimulated in the second "braking" task (MS/NS). The waveforms on the right depict average gamma/beta power for go trials (black), stop trials (red), successful stop trials (blue), and failed stop trials (green) and were used for selection of the experimental site depicted on the left. The shaded areas represent the SEM. The dotted box shows one or more classic stopping signatures for each subject. These plots were used to make the electrode selection for the stimulation experiment.

involved with seizure onset were excluded from all further analysis. All electrodes with $>10 \mathrm{~dB}$ of noise in the $60 \mathrm{~Hz}$ band were also excluded.

ECoG analysis. ECoG data were analyzed using custom scripts in MATLAB 2010b (The MathWorks). After being imported into MATLAB, the data were referenced by subtracting the average of all nonspiking channels from each individual channel. The data were then spliced into epochs ranging from -1000 to $3000 \mathrm{~ms}$ in relation to the event in question (Go stimulus for the Go vs Stop comparison, stop signal for the successful vs failed stop comparison). Artifacts were automatically detected using kurtosis and epoch-data probability distributions. Cutoff values were $\pm 5 \mathrm{SD}$ for both criteria. Analytic amplitudes for both power bands (beta: $13-30 \mathrm{~Hz}$; gamma: $30-200 \mathrm{~Hz}$ ) were generated using the absolute values of a Hilbert transform of the band-pass-filtered data (two-way least-squares finite impulse response filters). The values were baseline corrected to a time period $500 \mathrm{~ms}$ before the Go signal. These values were then averaged for each trial type individually and plotted as the percent change compared with baseline to identify stopping-related signatures. The resulting time series were filtered for display purposes using a Savitzky-Golay polynomial least-squares filter (second order, frame size: 255 samples).

$1 \mathrm{sec}$

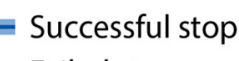

Failed stop

The full-beta spectrum ERSP plots (Fig. 3) were generated in a similar fashion: 20 linearly spaced center frequencies ranging from 13 to $30 \mathrm{~Hz}$ were preprocessed individually as described for the average-band power analyses described above [i.e., the data were individually filtered around each of the center frequencies (SD: $0.5 \mathrm{~Hz}$ ), and then converted to analytic amplitudes using the absolute values of the Hilbert transform of the band-passfiltered data]. The $z$-transformed values were tested for significance using a cluster-based false discovery rate thresholding procedure (cluster-threshold: $p<0.05$; overall threshold: $p<0.05)$.

Braking task ("MS/NS"). Subjects were instructed to perform a task similar to the localizer task (here called MS blocks), however, with intermittent blocks of a simple Go task in which no stop signals occurred (NS blocks). MS/NS blocks changed every 10 trials and were announced by an instruction screen displaying the respective condition (Fig. $1 B$ ). In addition, green (NS) and red (MS) squares around the stimuli were displayed on the screen in every block to remind subjects about the current block condition they were in. For the MS blocks, adaptive staircases were again used to adjust the SSD online, with the initial estimate carried over from the localizer session using the simple stopping task. Trial timing was identical to the localizer task. Subject S1 performed 400 trials (no control site); subjects S2 and S3 performed 800 trials ( 400 per site; i.e., experimental and control site); subject $\mathrm{S} 4$ performed three separate sessions on consecutive days $(600,800$, and 800 trials). A break was given every 50 trials.

Stimulation. Stimulation was delivered on Go trials in both the MS and NS condition using a Grass S88X with a stimulus isolation unit. On each trial, stimulation consisted of two 500 $\mathrm{ms}, 10 \mathrm{~mA}$ balanced square-wave pulses with an interpulse interval of $30 \mathrm{~ms}$.

We used two different conditions with regard to stimulation timing. One was time locked to the appearance of the Go stimulus (50 ms following the onset of the arrow) and the other one was time locked to an online estimate closely preceding the time of expected response emission. The stimulus-locked stimulation time point was added in case the RT forecast for the responselocked stimulation failed to generate enough valid trials. Because there were enough response-locked trials and because our predictions derived from a prior report pointing to response-locked activity (Swann et al., 2013), the stimulus-locked stimulation is not discussed further. The RT forecast for the response-locked stimulation condition was based on a trial-by-trial update of MS/NS GoRT. We delivered stimulation $50 \mathrm{~ms}$ (S1) or $100 \mathrm{~ms}$ (S2-4) before that estimate. We changed from 50 to 100 $\mathrm{ms}$ after we realized that too many trials might be lost by stimulation occurring after the button press given the inaccuracy in the RT forecasting method. The GoRT estimate was updated on every trial using the last 10 valid Go trials in the respective condition. The average stimulation times are shown in Table 1. Trials in which a response was emitted before stimulation occurred were dismissed from further analysis.

Stimulation timing conditions (no stimulation, stimulus-locked, response-locked) alternated on a trial-by-trial basis (the order was unknown to both subject and experimenter). Subject S1 only received experimental-site stimulation over rIFC. In subjects S2 and S3, stimula- 

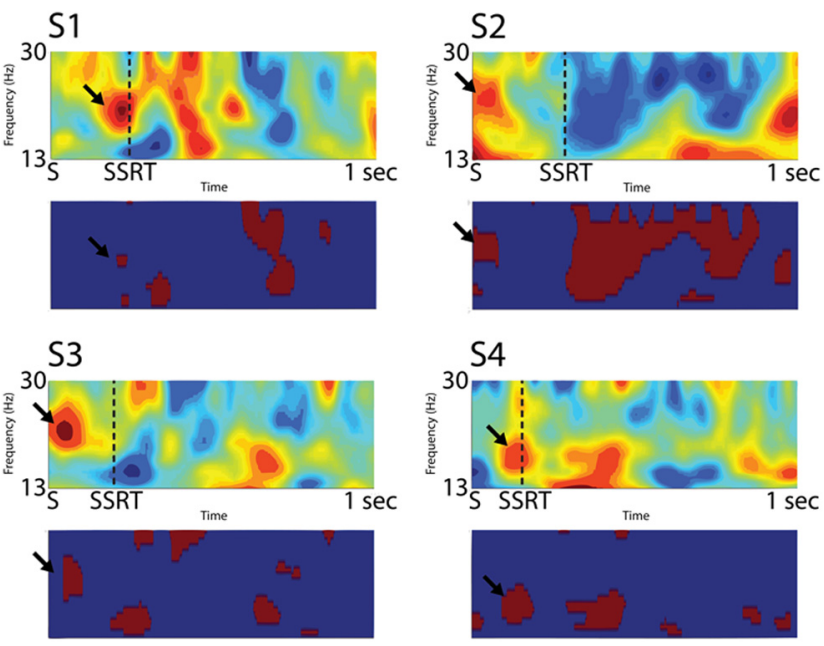

Figure 3. Full beta-spectrum ECOG data from the localizer SST in all four patients. Because only one subject showed the beta signature of interest in the rapidly generated plots in Figure 2 (required for selection during patient testing), we subsequently computed the full-spectrum beta ERSP plots for the successful versus failed stop trial comparison time locked to the stop signal (S). The figures show one of the two bipolar experimental stimulation sites (Fig. 2) that had the most clear-cut signature. The upper plots for each patient show the ERSP $z$-values for the contrast (successful minus failed stop trials), the lower plots denote FDR-corrected significance at $p<0.05$ with a cluster threshold of $p<0.05$. Red denotes significant clusters, blue areas are nonsignificant.

tion sites were switched during every break in the MS/NS experiment (i.e., every 50 trials) between rIFC and control site. Subject S4 had a temporal lobe control site in each session; however, because all sites were subsequently resected because of pathology, data from these sites were omitted.

$R T$ analysis. For the localizer and MS/NS task, SSRTs were calculated using the mean method (Verbruggen and Logan, 2009). For the MS/NS task, RT change was quantified on a block-by-block basis by subtracting mean GoRT on trials without stimulation (SHAM) from the mean GoRT in the stimulation conditions (STIM) separately for each MS or NS condition in each block (block refers to a block of 50 trials; subjects took a break every 50 trials). This way, we ended up with a blockwise quantification of slowing, with positive numbers indicating relative slowing (in milliseconds) induced by stimulation and negative numbers indicating stimulation-induced speeding. This RT change was averaged across blocks and was statistically tested for deviations from zero (zero representing the null hypothesis of no change in RT induced by stimulation) using group-label switching Monte Carlo analyses (10,000 iterations).

\section{Results}

\section{Localizer task}

All four subjects showed satisfactory behavioral performance compared with other patient studies using the SST (Swann et al., 2009) and compared with healthy population samples (i.e., reasonably fast Go-trial RT, stopping probabilities $\sim 0.5$, and stopsignal reaction times between 150 and 300 ms; Table 1).

\section{Braking task: individual subjects}

All four subjects showed satisfactory behavioral performance (Table 1), that is, slower MS than NS Go-trial RT, stopping probabilities mostly $\sim 0.5$; with one exception (subject $S 1 ; p=0.65$ ), and stop-signal reaction times very consistent with the localizer; with the same exception (S1). Error rates were very low and did not increase in the stimulation compared with the sham condition (Table 2).

In $\mathrm{S} 1, \mathrm{rIFC}$ stimulation induced slowing in the MS condition (76 ms, $p<0.0001$; all $p$-values two-sided unless otherwise specified), as well as the NS condition (30 ms, $p<0.01$ ), suggesting that rIFC stimulation enhances braking in general. However, slowing was much more pronounced in the MS than in the NS condition ( $p<0.05$; Fig. 4 ). To establish spatial specificity of this effect to the rIFC, for the next two subjects, stimulation alternated in a blockwise fashion every 50 trials between rIFC and a control site in lateral anterotemporal cortex.

In S2, a Monte Carlo ANOVA was performed for the factors CONDITION (MS vs NS) and SITE (rIFC vs control). There was a main effect of CONDITION $(p<0.01)$, no main effect of SITE $(p=0.5)$, and a marginally significant CONDITION $\times$ SITE interaction $(p=0.1)$. For MS trials, stimulation at rIFC induced slowing (159 ms, $p<0.0001)$, but not for NS trials $(p>0.4)$, and this difference was significant $(p<0.01)$. Against expectations, however, stimulation of the control site did induce slowing in both task conditions as well (MS: $89 \mathrm{~ms}, p<0.05$, NS: $62 \mathrm{~ms}, p<$ $0.05)$. Furthermore, slowing was not significantly increased for rIFC compared with the control site in either the MS (although this was a $70 \mathrm{~ms}$ difference, $p=0.2$, two-sided) or NS condition (32 ms, $p>0.3$ ). This discrepant pattern at the control site was clarified by further analysis: The control site had stopping-related ECoG localizer activity (Fig. 5), suggesting that it may have been activated by the auditory stop signal and may thus be connected to the stopping network. Although the control site was some distance from primary auditory cortex, fMRI studies do show that wider temporal cortex is activated when stop signals are presented in the auditory domain (Aron and Poldrack, 2006). Therefore, we collected data from S3 after verifying that the control site did not show any stopping-related localizer activity.

In S3, ANOVA revealed a main effect of SITE ( $p=0.01)$, no main effect of CONDITION ( $p>0.3)$, and, importantly, a significant CONDITION $\times$ SITE interaction $(p<0.01)$. For MS trials, and replicating S1 and S2, rIFC stimulation induced slowing (74 ms, $p<0.0001$ ). Importantly, there was no slowing at the control site ( $3 \mathrm{~ms}, p>0.9)$ and, moreover, the slowing was greater for rIFC versus control site $(71 \mathrm{~ms}, p<0.01)$. For NS trials, there was also slowing, now at both sites (experimental: 23 ms, $p<0.05$, control: $29 \mathrm{~ms}, p<0.0001$ ); however, there was no significant difference between sites $(6 \mathrm{~ms}, p>0.6)$. Again, slowing was greater for MS than NS in $\operatorname{rIFC}(p<0.01)$, but now, importantly, not the control site (n.s.).

In S4, we tested the within-subject reliability of stimulationinduced slowing by running three separate stimulation sessions on different days. Because much of temporal cortex was resected due to epileptiform activity, there was no valid control site. Again, rIFC stimulation induced slowing in the MS condition and this was very stable across sessions (Session 1: $69 \mathrm{~ms}, p<$ 0.01; Session 2: $73 \mathrm{~ms} ; p<0.05$, one-sided; Session 3: $69 \mathrm{~ms}, p<$ $0.05)$. There was no stimulation-induced slowing for the NS condition in any session (Session 1: $14 \mathrm{~ms}, p>0.2$; Session 2: $18 \mathrm{~ms}$, $p>0.1$; Session 3: $-1 \mathrm{~ms}, p>0.9$ ). Again, slowing was greater for MS vs NS, stably across sessions (Session 1: $p<0.001$; Session 2: $p<0.01$; Session 3: $p<0.001$; Fig. 6).

\section{Braking task: further analysis}

Although our data are consistent with stimulation augmenting a rIFC-mediated, context-dependent brake (i.e., stimulation induced more slowing for MS than NS), it is possible that such stimulation merely altered the Go process somehow more for MS than NS given that there was already a baseline RT difference (i.e., in the absence of stimulation) between these conditions. Accordingly, we performed a new analysis in which the stimulationinduced RT effects were normalized by the baseline RT in each MS or NS condition; that is, we calculated $100^{\star}($ MS_Stim - 
Table 1. Behavioral data from the two tasks

\begin{tabular}{|c|c|c|c|c|c|c|c|c|c|c|c|c|}
\hline \multicolumn{13}{|c|}{ Localizer task (SST) } \\
\hline & \multicolumn{2}{|c|}{ Correct Go RT (ms) } & \multicolumn{2}{|c|}{ Direction errors (\%) } & \multicolumn{3}{|c|}{ Omission errors (\%) } & \multicolumn{2}{|c|}{ FSRT (ms) } & $\mathrm{p}$ (inh) \% & SSD (ms) & SSRT (ms) \\
\hline S1 & \multicolumn{2}{|l|}{590} & \multicolumn{2}{|c|}{0.4} & \multicolumn{3}{|c|}{0} & \multicolumn{2}{|c|}{486} & 48.3 & 346 & 243 \\
\hline S2 & \multicolumn{2}{|l|}{721} & \multicolumn{2}{|c|}{0.9} & \multicolumn{3}{|c|}{1.4} & \multicolumn{2}{|c|}{568} & 49.4 & 436 & 285 \\
\hline S3 & \multicolumn{2}{|l|}{780} & \multicolumn{2}{|l|}{0} & \multicolumn{3}{|c|}{0.9} & \multicolumn{2}{|c|}{638} & 50 & 577 & 203 \\
\hline S4 & \multicolumn{2}{|l|}{901} & \multicolumn{2}{|l|}{0} & \multicolumn{3}{|c|}{2.5} & \multicolumn{2}{|c|}{766} & 52.7 & 745 & 156 \\
\hline \multicolumn{13}{|c|}{ Stimulation task (MS/NS) } \\
\hline \multirow[b]{2}{*}{ Condition } & \multicolumn{2}{|c|}{$\begin{array}{l}\text { Correct Go RT } \\
\text { (ms) }\end{array}$} & \multicolumn{2}{|c|}{$\begin{array}{l}\text { Stimulation } \\
\text { timing* }^{*}(\mathrm{~ms})\end{array}$} & $\begin{array}{l}\text { Direc } \\
(\%)\end{array}$ & & $\begin{array}{l}\text { Omis } \\
(\%)\end{array}$ & rors & FSRT (ms) & $\mathrm{p}$ (inh) $(\%)$ & SSD (ms) & SSRT (ms) \\
\hline & MS & NS & MS & NS & MS & NS & MS & NS & MS & MS & MS & MS \\
\hline S1 & 880 & 513 & 738 & 429 & 0.0 & 0.0 & 1.9 & 0.0 & 789 & 65 & 558 & 323 \\
\hline S2 & 801 & 666 & 582 & 497 & 2.2 & 0.0 & 1.1 & 0.0 & 725 & 48 & 555 & 246 \\
\hline S3 & 674 & 512 & 559 & 402 & 0.0 & 0.8 & 0.0 & 1.6 & 595 & 50 & 485 & 189 \\
\hline S4 (day 1) & 755 & 470 & 624 & 345 & 0.0 & 0.0 & 0.0 & 0.0 & 673 & 51 & 583 & 172 \\
\hline S4 (day 2) & 870 & 455 & 702 & 335 & 0.0 & 0.0 & 0.0 & 0.0 & 742 & 51 & 691 & 179 \\
\hline S4 (day 3) & 631 & 473 & 479 & 345 & 0.0 & 1.5 & 0.0 & 0.0 & 543 & 48 & 454 & 177 \\
\hline
\end{tabular}

FSRT, Failed stop trial reaction time.

*Average stimulation time that depended on the trial-by-trial forecast of GoRT.

Table 2. Absolute number of direction errors (wrong key press in response to the go signal) on go trials in the MS/NS experiment listed per task condition and stimulation condition

\begin{tabular}{|c|c|c|c|c|}
\hline \multirow{3}{*}{$\begin{array}{l}\text { Stimulation } \\
\text { condition }\end{array}$} & \multicolumn{4}{|c|}{ Direction errors (number) } \\
\hline & \multicolumn{2}{|c|}{ STIM } & \multicolumn{2}{|c|}{ SHAM } \\
\hline & MS & NS & MS & NS \\
\hline S1 & 0 & 0 & 0 & 0 \\
\hline S2 & 1 & 1 & 2 & 0 \\
\hline S3 & 0 & 1 & 0 & 1 \\
\hline S4 (day 1) & 0 & 0 & 0 & 0 \\
\hline S4 (day 2) & 0 & 1 & 0 & 0 \\
\hline S4 (day 3) & 0 & 1 & 2 & 2 \\
\hline
\end{tabular}

Stimulation did not elicit a higher number of direction errors. STIM, Stimulation; SHAM, no stimulation.
S1

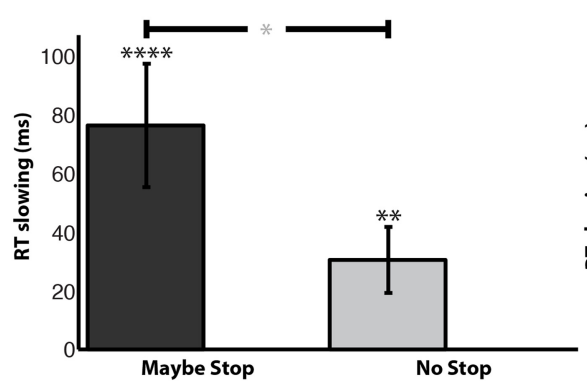

S3

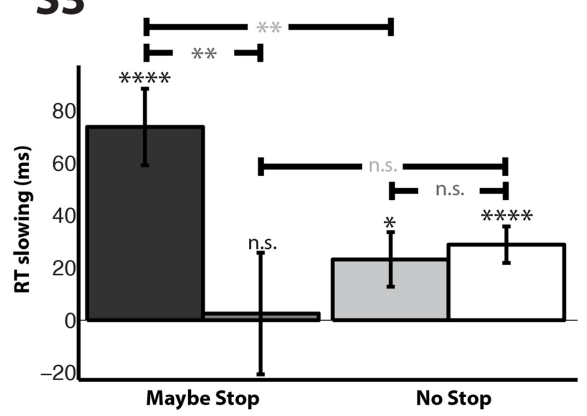

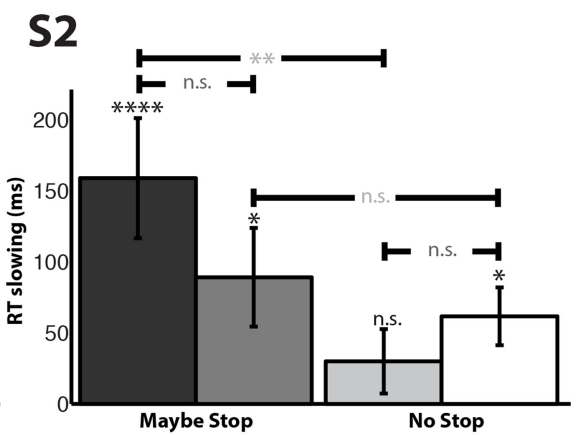

Maybe Stop

Figure legend

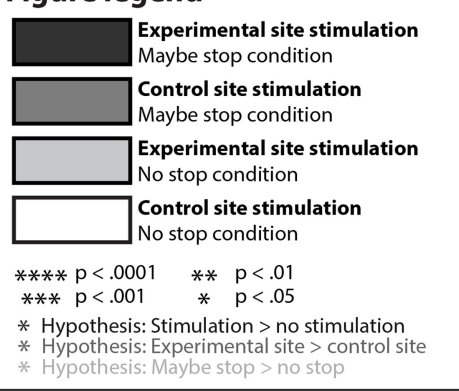

Figure 4. Stimulation-induced RT slowing for subjects S1-S3. Bars represent average of stimulation-induced changes in RT (go trial RT on stimulation trials minus go trial RT on sham trials quantified for each block of 50 trials individually and subsequently averaged for each subject). Zero represents no change in RT induced by stimulation; positive values indicate slowing and negative values indicate speeding. Significance stars are color coded according to hypotheses and result from randomized group-label switching Monte Carlo simulated ttests.
MS_Sham)/MS_Sham and $100^{*}($ NS_Stim - NS_Sham)/NS_Sham, respectively, instead of the initial quantification of MS Stim - MS_Sham and NS_Stim - NS_Sham. We then tested these percentage changes in stimulation-induced RT differences between MS and NS for significance as we did for the initial analysis.

Stimulation-induced differences in braking were still numerically greater in the MS compared with the NS condition in all six sessions (S1, S2, S3, and all three sessions for S4), as for the initial analysis. These effects remained significant in 4 of 6 of the sessions, but not in $S 1(p=0.51)$ and not in Session 2 of $S 4(p=$ 0.18 , Table 3 ). Perhaps unsurprisingly, these were the two sessions with the largest nonstimulation MS vs NS differences; that is, the sessions in which subjects already exerted the most motor caution in the absence of stimulation. This raises the interesting prospect that if a subject already brakes to a large amount, stimulation will not induce a large amount of additional slowing, yet if only a minor amount of motor caution is exerted to begin with, stimulation will have larger effects. Crucially, this predicts a negative correlation between the amount of stimulationinduced MS versus NS slowing as quantified by the percent-change measurement described above and the braking exerted by the subjects in the absence of stimulation (the MS vs NS RT difference in the sham condition). We tested this correlation across the six sessions and indeed found a highly negative correlation $(r=$ $-0.94, p=0.0167$, Spearman correlation; Fig. 7).

\section{Discussion}

For each of four subjects, we localized an rIFC site having an electrophysiological signature of inhibitory control for outright stopping and then, in a subsequent session, we electrically stimulated that site during a braking task. In all subjects, rIFC 
stimulation slowed down responding, and more so in the braking (MS) than nonbraking (NS) context. Critically, in S3, in whom the control site was not connected to the "stopping network," stopping-network, rIFC stimulation increased braking more than control-site stimulation (S2 showed the same numerical pattern). Stimulation-induced slowing for rIFC was highly reliable across and within subjects (Figs. 4, 6). This is the first demonstration of behavioral change induced by a single pair of prefrontal DES pulses time locked to ongoing behavior in humans. Moreover, this was done in double-blind fashion (neither the experimenter nor the patient was aware of whether stimulation was delivered on a given trial or not, or which site was being stimulated) using a functionally localized experimental site, a control site, and computer-controlled stimulation with millisecond precision.

Stimulation elongated RT in the braking context (MS) more than in the nonbraking context (NS). Given that the timing of stimulation was more than half a second after the Go stimulus and temporally much closer to the movement, this is not consistent with a disruption of the decision process (of which button to press); also, we note that stimulation did not affect accuracy (Table 2). It is also unlikely that rIFC stimulation merely affected motor initiation because, again, slowing was greater for MS than NS (when there was initiation in both conditions) and this remained true in four of six sessions even when taking into account the baseline (nonstimulation) slowing. There was also a striking correlation: stimulation induced greater slowing for those subjects who did not slow down much without stimulation. This is evidence against stimulation merely interfering with the movement itself and is more consistent with an augmentation of an endogenously activated brake provided that it is not already maximally engaged (i.e., we suppose that the rIFC-based braking system was maximally active at non-stimulation baseline in some patients, and DES could not affect it - a form of saturation). One might wonder whether rIFC stimulation disrupted the release of a brake rather than augmenting a brake. However, that account does not explain the fact that rIFC is more active for successful than failed outright stopping in this and another study before SSRT (Swann et al., 2009) and that the rIFC is critical for outright stopping (Aron et al., 2003; Chambers et al., 2006). Instead, we argue that rIFC stimulation enhanced a rIFC-mediated brake.

Our results are relevant to debates about rIFC's role in inhibitory control. By showing that artificially activating a node of the brain's stopping network slows down responding without an explicit stop signal, the results argue against the view that the rIFC is merely an attentional/salience detector (Hampshire et al., 2010; Sharp et al., 2010) because here it was causally important for a form of inhibitory control even in the absence of any signal. Our results are also inconsistent with the view that rIFC is merely an attentional/contextual monitor (Stuss and Alexander, 2007; Munakata et al., 2011; Chatham et al., 2012). Although stimulation of an attentional monitor would lead to a better or worse detection of a stop signal on Stop trials, here we are only examining RT on Go trials. We cannot see how altering an attentional monitor would affect Go trial RTs unless that monitor is tightly coupled

\section{S2: Gamma-band power Locked to go stimulus}

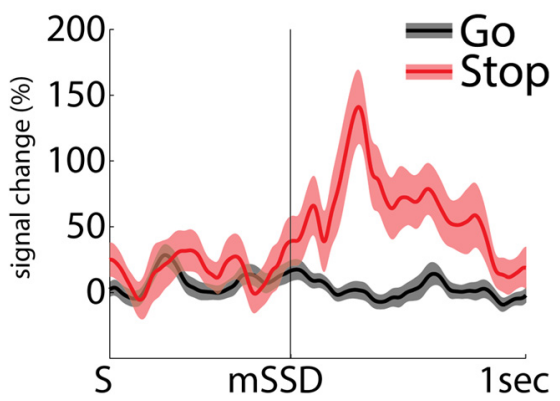

Figure 5. The temporal lobe control site in $\$ 2$ showed enlarged gamma-band activity on stop trials compared with go trials after the stop signal presentation (potentially due to auditory processing of the stop signal).

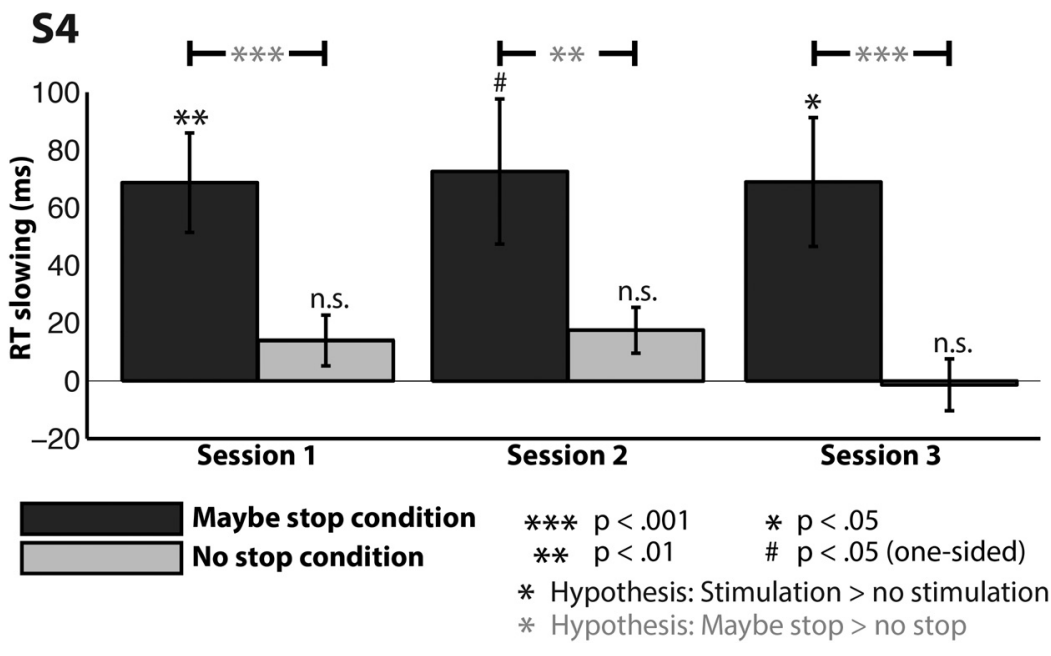

Figure 6. Stimulation-induced RT slowing in $\mathrm{S4}$ is stable across sessions. See Figure 4 legend for figure information. an inhibitory control system. Therefore although this does not rule out the possibility that rIFC is involved in both attentional monitoring and motor inhibition, it refutes the "pure" attentional monitoring account. What also refutes this account is the observation that rIFC ECoG activity tracked the time of the response much better than the estimated time of the stop signal, which is consistent with a brake (Swann et al., 2013). Together, these results argue for the view that rIFC is tightly integrated within a network for inhibitory control of action tendencies, which can lead to braking, motor pauses, or indeed outright stopping (Aron et al., 2004; Chambers et al., 2009; Neubert et al., 2010; Levy and Wagner, 2011; Wiecki and Frank, 2013). Although rIFC is integrated with presupplementary motor area and the subthalamic nucleus (STN) for outright stopping, it is not yet clear how rIFC implements the putative braking function via a wider network; this could be via striatum, STN, or premotor cortex/M1.

Our results also show how DES can provide key information about the causal role of brain networks in human cognitive function. One other potential method to test a causal role is transcranial magnetic stimulation (TMS). There have been several studies using TMS with stopping paradigms (Chambers et al., 2006; Chambers et al., 2007; Verbruggen et al., 2010); however, these were offline stimulation, not event related, and they did not so much investigate braking as outright stopping. This is a crucial 
Table 3. Comparison of the differential effects of stimulation on MS versus NS trials for the two different methods of quantification (raw-stimulation-induced RT difference versus percent change)

\begin{tabular}{|c|c|c|c|c|c|c|c|c|c|c|c|c|}
\hline & \multicolumn{2}{|l|}{ S1 } & \multicolumn{2}{|l|}{ S2 } & \multicolumn{2}{|l|}{ S3 } & \multicolumn{2}{|c|}{ S4 (day 1) } & \multicolumn{2}{|c|}{ S4 (day 2) } & \multicolumn{2}{|c|}{ S4 (day 3) } \\
\hline & Diff & $p$ & Diff & $p$ & Diff & $p$ & Diff & $p$ & Diff & $p$ & Diff & $p$ \\
\hline \multicolumn{13}{|l|}{ rIFC } \\
\hline Raw RT & 45.79 & 0.04 & 128.96 & 0.01 & 73.76 & 0.00 & 54.68 & 0.01 & 55.04 & 0.01 & 70.23 & 0.00 \\
\hline$\%$ & 2.17 & 0.51 & 14.63 & 0.03 & 7.00 & 0.02 & 6.01 & 0.05 & 4.45 & 0.18 & 10.42 & 0.01 \\
\hline \multicolumn{13}{|l|}{ Control } \\
\hline Raw RT & & & 27.48 & 0.51 & 2.54 & 0.92 & & & & & & \\
\hline$\%$ & & & 1.58 & 0.80 & -4.30 & 0.15 & & & & & & \\
\hline \multicolumn{13}{|l|}{ ANOVA } \\
\hline \multicolumn{13}{|l|}{ Site } \\
\hline Raw RT & & & & 0.53 & & 0.01 & & & & & & \\
\hline$\%$ & & & & 0.86 & & 0.04 & & & & & & \\
\hline \multicolumn{13}{|l|}{ MSNS } \\
\hline Raw RT & & & & 0.01 & & 0.36 & & & & & & \\
\hline$\%$ & & & & 0.05 & & 0.54 & & & & & & \\
\hline \multicolumn{13}{|l|}{ Inter. } \\
\hline Raw RT & & & & 0.10 & & 0.00 & & & & & & \\
\hline$\%$ & & & & 0.13 & & 0.01 & & & & & & \\
\hline
\end{tabular}

Diff, Differential effect of stimulation.

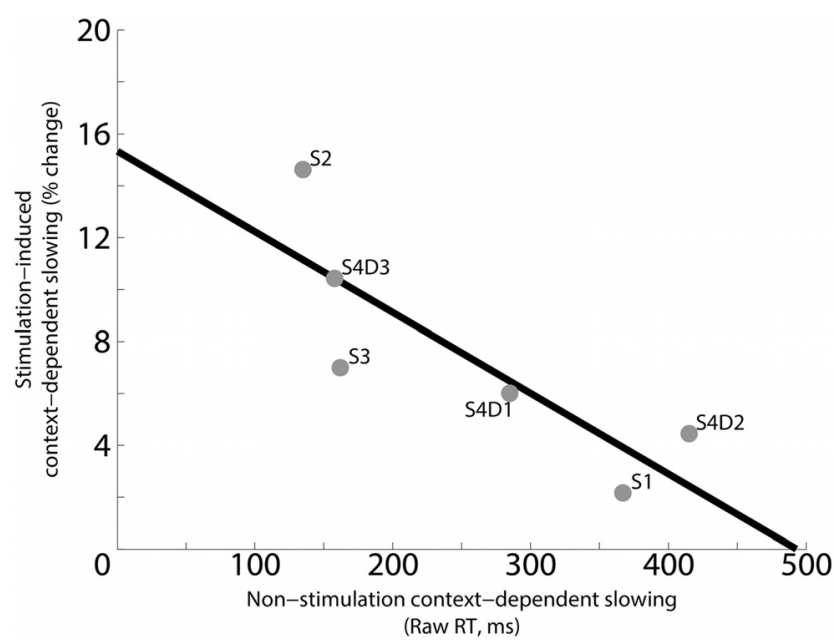

Figure 7. The less subjects slowed down in the absence of stimulation, the more braking was induced by rIFC-stimulation. The correlation is between the amount of stimulation-induced context-dependent (MS vs NS) braking (normalized to percent change from NS GoRT without stimulation) and the amount of context-dependent (MS vs NS) braking as implemented by the subjects in the absence of stimulation.

distinction because a causal implication of rIFC in braking implies that it can be recruited by endogenously triggered control (intentions) rather than merely by external stop signals. DES provides some notable methodological advances compared with TMS. To provide appropriate temporal resolution, TMS would have to be done concurrent with task performance (i.e., eventrelated). However, event-related TMS of rIFC at a level sufficient to affect behavior is very uncomfortable given the proximity of facial muscles. It is also difficult for such TMS studies to reach our current standard of fully double-blind experimental control. This is because facial sensation is generated by such TMS, making sham stimulation difficult. Further, the experimenter has to physically hold a coil at the stimulation location, which also makes control-site blinding difficult. Another difference between our form of DES and TMS is that the latter has a mass action effect that usually results in disruption rather than facilitation of behavior, whereas here the event-related DES appears to be facilitatory. We used a novel form of DES that overcomes the limitations of standard DES, which typically entails high-frequency $(60 \mathrm{~Hz})$ stimulation for durations $>1 \mathrm{~s}$ and often lacks double-blinding and other experimental controls (Borchers et al., 2012; Filevich et al., 2012). In short, we have exploited the merits of DES over TMS in the current paradigm to provide a fully double-blinded, shamand location-controlled demonstration of the rIFC's role in braking. However, we note that DES is invasive and used exclusively on populations with neurological conditions. We regard the current results as a starting point for further experiments, including using various forms of TMS in healthy subject populations.

We delivered stimulation at rIFC in each subject and, in some subjects, we compared it with control-site stimulation in the temporal lobe. We did not, however, compare rIFC stimulation with many other possible prefrontal and premotor stimulation sites because it is impractical for patients to participate in more stimulation conditions. However, several considerations do suggest the likely specificity to the rIFC. First, we functionally localized a rIFC region based on a stop-signal electrophysiological signature of inhibitory control (gamma or beta power increase). Such outright stopping signatures are mostly seen in the posterior rIFC/ premotor region (Swann et al., 2009, 2012) and not widely throughout lateral prefrontal cortex (also see fMRI meta-analysis by Levy and Wagner, 2011). Second, our observation of a striking effect of stimulation at loci that elicit an inhibitory control signature is also consistent with a study in monkeys (Sasaki et al., 1989). In that study, Sasaki et al. performed a much wider stimulation mapping of lateral prefrontal cortex, showing that stimulation of loci with an electrophysiological signature of inhibitory control (and not other regions without such a signature) was most effective in delaying or canceling behavior. Regarding specificity, one might wonder if our stimulation had its effects via general current spread rather than a specific effect at rIFC. Although stimulation does produce evoked responses in adjacent regions of cortex (Borchers et al., 2012), it is unlikely that those evoked responses themselves are systematic and strong enough, across subjects, to produce the same amount of elongation of RT that we found here, especially in the MS condition. A recent review (Desmurget et al., 2013) made a compelling case that DES effects on behavior do not merely reflect an anarchic spread of current. A final consideration in favor of a specific effect at rIFC is that rIFC stimulation produced a significantly larger RT slowing for MS trials compared with NS trials in all subjects and sessions. Such MS trials are known to activate rIFC compared 
with NS trials and they do so in a rather locally specific manner (including rIFC and middle frontal gyrus) in both ECoG and fMRI (Swann et al., 2012, 2013), with activity peaking around the time we stimulated in the current experiment (Swann et al., 2013). Therefore, the behavioral effects of stimulation seen here are likely specific to rIFC itself and would probably not occur with stimulation of other prefrontal regions, but this is a topic for future investigation.

In summary, using a novel application of DES with doubleblind control and precise task-based stimulation, we provide causal evidence for a rIFC-mediated braking function. This argues strongly for classic views that lateral prefrontal cortex is important for implementing inhibitory action control (Iversen and Mishkin, 1970; Sasaki et al., 1989; Dias et al., 1996; Fuster, 1997; Knight et al., 1999) and against the view that rIFC is merely an attentional detector/monitor (Stuss and Alexander, 2007; Munakata et al., 2011; Chatham et al., 2012). The findings also extend this inhibitory control function beyond externally driven action cancellation to forms of self-control that are internally generated according to an individual's goals (Brass and Haggard, 2007; Aron, 2011). This may help to explain why this brain system has been implicated in various disorders of impulse control that are not simply characterized by deficits of phasic stimulus-driven inhibitory control, but which might instead involve internally generated control, done partially or completely, and perhaps repeatedly over a tonic interval in relation to urges (Robbins, 2007; Chambers et al., 2009; Marsh et al., 2009; Berman et al., 2012; Whelan et al., 2012). The results also provide key insights into the parameters for electrical stimulation in human prefrontal cortex that influence cognitive control (Pesaran et al., 2006).

\section{References}

Amunts K, Schleicher A, Bürgel U, Mohlberg H, Uylings HB, Zilles K (1999) Broca's region revisited: cytoarchitecture and intersubject variability. J Comp Neurol 412:319-341. CrossRef Medline

Aron AR (2011) From reactive to proactive and selective control: developing a richer model for stopping inappropriate responses. Biol Psychiatry 69:e55-e68. CrossRef Medline

Aron AR, Poldrack RA (2006) Cortical and subcortical contributions to Stop signal response inhbition: role of the subthalamic nucleus. J Neurosci 26:2424-2433. CrossRef Medline

Aron AR, Fletcher PC, Bullmore ET, Sahakian BJ, Robbins TW (2003) Stopsignal inhibition disrupted by damage to right inferior frontal gyrus in humans. Nat Neurosci 6:115-116. CrossRef Medline

Aron AR, Robbins TW, Poldrack RA (2004) Inhibition and the right inferior frontal cortex. Trends Cogn Sci 8:170-177. CrossRef Medline

Aron AR, Behrens TE, Smith S, Frank MJ, Poldrack RA (2007) Triangulating a cognitive control network using diffusion-weighted magnetic resonance imaging (MRI) and functional MRI. J Neurosci 27:3743-3752. CrossRef Medline

Berman BD, Horovitz SG, Morel B, Hallett M (2012) Neural correlates of blink suppression and the buildup of a natural bodily urge. Neuroimage 59:1441-1450. CrossRef Medline

Borchers S, Himmelbach M, Logothetis N, Karnath HO (2012) Direct electrical stimulation of human cortex-the gold standard for mapping brain functions? Nat Rev Neurosci 13:63-70. CrossRef Medline

Brass M, Haggard P (2007) To do or not to do: the neural signature of self-control. J Neurosci 27:9141-9145. CrossRef Medline

Chambers CD, Bellgrove MA, Stokes MG, Henderson TR, Garavan H, Robertson IH, Morris AP, Mattingley JB (2006) Executive "brake failure" following deactivation of human frontal lobe. J Cogn Neurosci 18:444455. Medline

Chambers CD, Bellgrove MA, Gould IC, English T, Garavan H, McNaught E, Kamke M, Mattingley JB (2007) Dissociable mechanisms of cognitive control in prefrontal and premotor cortex. J Neurophysiol 98:3638-3647. CrossRef Medline

Chambers CD, Garavan H, Bellgrove MA (2009) Insights into the neural basis of response inhibition from cognitive and clinical neuroscience. Neurosci Biobehav Rev 33:631-646. CrossRef Medline

Chatham CH, Claus ED, Kim A, Curran T, Banich MT, Munakata Y (2012) Cognitive control reflects context monitoring, not motoric stopping, in response inhibition. PLoS One 7:e31546. CrossRef Medline

Chikazoe J, Jimura K, Hirose S, Yamashita K, Miyashita Y, Konishi S (2009) Preparation to inhibit a response complements response inhibition during performance of a stop-signal task. J Neurosci 29:15870-15877. CrossRef Medline

Chiu YC, Aron AR (2013) Unconsciously triggered response inhibition requires an executive setting. J Exp Psychol Gen, in press. Medline

Conner CR, Ellmore TM, Pieters TA, DiSano MA, Tandon N (2011) Variability of the relationship between electrophysiology and BOLD-fMRI across cortical regions in humans. J Neurosci 31:12855-12865. CrossRef Medline

Desmurget M, Song Z, Mottolese C, Sirigu A (2013) Re-establishing the merits of electrical brain stimulation. Trends Cogn Sci 17:442-449. CrossRef Medline

Dias R, Robbins TW, Roberts AC (1996) Dissociation in prefrontal cortex of affective and attentional shifts. Nature 380:69-72. CrossRef Medline

Filevich E, Kühn S, Haggard P (2012) Negative motor phenomena in cortical stimulation: implications for inhibitory control of human action. Cortex 48:1251-1261. CrossRef Medline

Forstmann BU, Keuken MC, Jahfari S, Bazin PL, Neumann J, Schäfer A, Anwander A, Turner R (2012) Cortico-subthalamic white matter tract strength predicts interindividual efficacy in stopping a motor response. Neuroimage 60:370-375. CrossRef Medline

Fuster JM (1997) The prefrontal cortex: anatomy, physiology and neuropsychology of the frontal lobe. Philadelphia: Lippincott-Raven.

Hampshire A, Chamberlain SR, Monti MM, Duncan J, Owen AM (2010) The role of the right inferior frontal gyrus: inhibition and attentional control. Neuroimage 50:1313-1319. CrossRef Medline

Iversen SD, Mishkin M (1970) Perseverative interference in monkeys following selective lesions of the inferior prefrontal convexity. Exp Brain Res 11:376-386. Medline

Jahfari S, Stinear CM, Claffey M, Verbruggen F, Aron AR (2010) Responding with restraint: what are the neurocognitive mechanisms? J Cogn Neurosci 22:1479-1492. CrossRef Medline

Jahfari S, Verbruggen F, Frank MJ, Waldorp LJ, Colzato L, Ridderinkhof KR, Forstmann BU (2012) How preparation changes the need for top-down control of the basal ganglia when inhibiting premature actions. J Neurosci 32:10870-10878. CrossRef Medline

Knight RT, Staines WR, Swick D, Chao LL (1999) Prefrontal cortex regulates inhibition and excitation in distributed neural networks. Acta Psychologica 101:159-178. CrossRef Medline

Levy BJ, Wagner AD (2011) Cognitive control and right ventrolateral prefrontal cortex: reflexive reorienting, motor inhibition, and action updating. Ann N Y Acad Sci 1224:40-62. CrossRef Medline

Lüders HO, Dinner DS, Morris HH, Wyllie E, Comair YG (1995) Cortical electrical stimulation in humans: the negative motor areas. Adv Neurol 67:115-129. Medline

Marsh R, Maia TV, Peterson BS (2009) Functional disturbances within frontostriatal circuits across multiple childhood psychopathologies. Am J Psychiatr 166:664-674. CrossRef Medline

Munakata Y, Herd SA, Chatham CH, Depue BE, Banich MT, O'Reilly RC (2011) A unified framework for inhibitory control. Trends Cogn Sci 15: 453-459. CrossRef Medline

Neubert FX, Mars RB, Buch ER, Olivier E, Rushworth MF (2010) Cortical and subcortical interactions during action reprogramming and their related white matter pathways. Proc Natl Acad Sci U S A 107:13240-13245. CrossRef Medline

Pesaran B, Musallam S, Andersen RA (2006) Cognitive neural prosthetics. Curr Biol 16:R77-R80. CrossRef Medline

Robbins TW (2007) Shifting and stopping: fronto-striatal substrates, neurochemical modulation and clinical implications. Philos Trans R Soc Lond B Biol Sci 362:917-932. CrossRef Medline

Sasaki K, Gemba H, Tsujimoto T (1989) Suppression of visually initiated hand movement by stimulation of the prefrontal cortex in the monkey. Brain Res 495:100-107. CrossRef Medline

Sharp DJ, Bonnelle V, De Boissezon X, Beckmann CF, James SG, Patel MC, Mehta MA (2010) Distinct frontal systems for response inhibition, at- 
tentional capture, and error processing. Proc Natl Acad Sci U S A 107: 6106-6111. CrossRef Medline

Stuss DT, Alexander MP (2007) Is there a dysexecutive syndrome? Phil Trans R Soc B 362:901-915. CrossRef

Swann NC, Tandon N, Pieters TA, Aron AR (2013) Intracranial electroencephalography reveals different temporal profiles for dorsal- and ventrolateral prefrontal cortex in preparing to stop action. Cereb Cortex 23: 2479-2488. CrossRef Medline

Swann NC, Cai W, Conner CR, Pieters TA, Claffey MP, George JS, Aron AR, Tandon N (2012) Roles for the pre-supplementary motor area and the right inferior frontal gyrus in stopping action: electrophysiological responses and functional and structural connectivity. Neuroimage 59: 2860-2870. CrossRef Medline

Swann N, Tandon N, Canolty R, Ellmore TM, McEvoy LK, Dreyer S, DiSano M, Aron AR (2009) Intracranial EEG reveals a time- and frequencyspecific role for the right inferior frontal gyrus and primary motor cortex in stopping initiated responses. J Neurosci 29:12675-12685. CrossRef Medline

Verbruggen F, Logan GD (2009) Models of response inhibition in the stopsignal and stop-change paradigms. Neurosci Biobehav Rev 33:647-661. CrossRef Medline
Verbruggen F, Aron AR, Stevens MA, Chambers CD (2010) Theta burst stimulation dissociates attention and action updating in human inferior frontal cortex. Proc Natl Acad Sci U S A 107:13966-13971. CrossRef Medline

Whelan R, Conrod PJ, Poline JB, Lourdusamy A, Banaschewski T, Barker GJ, Bellgrove MA, Büchel C, Byrne M, Cummins TD, Fauth-Bühler M, Flor H, Gallinat J, Heinz A, Ittermann B, Mann K, Martinot JL, Lalor EC, Lathrop M, Loth E, Nees F, et al.; IMAGEN Consortium. (2012) Adolescent impulsivity phenotypes characterized by distinct brain networks. Nat Neurosci 15:920-925. CrossRef Medline

Wiecki TV, Frank MJ (2013) A computational model of inhibitory control in frontal cortex and basal ganglia. Psychol Rev 120:329-355. CrossRef Medline

Zandbelt BB, Bloemendaal M, Neggers SF, Kahn RS, Vink M (2013a) Expectations and violations: delineating the neural network of proactive inhibitory control. Hum Brain Mapp 34:2015-2024. Medline

Zandbelt BB, Bloemendaal M, Hoogendam JM, Kahn RS, Vink M (2013b) Transcranial magnetic stimulation and functional MRI reveal cortical and subcortical interactions during stop-signal response inhibition. J Cogn Neurosci 25:157-174. CrossRef Medline 